\title{
EMPIRICAL LIKELIHOOD FOR HIGH FREQUENCY DATA
}

\author{
LORENZO CAMPONOVO, YUKITOSHI MATSUSHITA, AND TAISUKE OTSU
}

\begin{abstract}
This paper introduces empirical likelihood methods for interval estimation and hypothesis testing on volatility measures in some high frequency data environments. We propose a modified empirical likelihood statistic that is asymptotically pivotal under infill asymptotics, where the number of high frequency observations in a fixed time interval increases to infinity. The proposed statistic is extended to be robust to the presence of jumps and microstructure noise. We also provide an empirical likelihood-based test to detect the presence of jumps. Furthermore, we study higher-order properties of a general family of nonparametric likelihood statistics and show that a particular statistic admits a Bartlett correction: a higher-order refinement to achieve better coverage or size properties. Simulation and a real data example illustrate the usefulness of our approach.
\end{abstract}

\section{INTRODUCTION}

Realized volatility and its related statistics have become standard tools to explore the behavior of high frequency financial data and to evaluate theoretical financial models including stochastic volatility models. This increase in popularity has been driven by recent developments in probability and statistical theory and by the increasing availability of high frequency financial data (see, Aït-Sahalia and Jacod, 2014, for a review).

By using infill asymptotics, where the number of high frequency observations in a fixed time interval (say, a day) increases to infinity, Jacod and Protter (1998) and BarndorffNielsen and Shephard (2002) established laws of large numbers and central limit theorems for realized volatility, which were later extended to more general setups and statistics by

The authors acknowledge helpful comments from an associate editor and anonymous referees. This research was partly supported by the JSPS KAKENHI Grant Number 26780133, 18K01541 (Matsushita), and the ERC Consolidator Grant (SNP 615882) (Otsu). 
Barndorff-Nielsen et al. (2006). Gonçalves and Meddahi (2009) studied second-order properties of the realized volatility statistic and its bootstrap counterpart. Furthermore, a variety of volatility estimation methods are developed to be robust to the presence of jumps (e.g., Barndorff-Nielsen, Shephard and Winkel, 2006, and Andersen, Dobrev and Schaumburg, 2012) and microstructure noise (e.g., Zhang, Mykland and Aït-Sahalia, 2005, Barndorff-Nielsen et al., 2008, and Jacod et al., 2009). There have also been several testing methods for the presence of jumps (e.g., Barndorff-Nielsen and Shephard, 2006, and Aït-Sahalia and Jacod, 2009a).

In this paper, we introduce empirical likelihood methods (see, Owen, 2001, for a review) for interval estimation and hypothesis testing on volatility measures in different high frequency data environments. In particular, based on estimating equations for the volatility measures, such as the integrated volatility, a modified empirical likelihood statistic is proposed and shown to be asymptotically pivotal under infill asymptotics. Our empirical likelihood approach is extended to be robust to the presence of jumps and microstructure noise. The proposed statistics share desirable properties with conventional empirical likelihood, such as being range preserving, being transformation respecting, and having a data decided shape for the confidence region. We also provide an empirical likelihoodbased test to detect the presence of jumps. Our empirical likelihood approach provides useful alternatives to the existing Wald-type inference methods and jump tests. This is illustrated by simulation studies and a real data example.

Another distinguishing feature of (conventional) empirical likelihood is that it admits a Bartlett correction: a higher-order refinement to achieve better coverage and size properties (DiCiccio, Hall and Romano, 1991). However, since empirical moments typically exhibit rather different limiting behaviors under infill asymptotics, empirical likelihood is 
not Bartlett correctable even for the constant volatility setup. In order to further investigate this issue, we consider a general class of nonparametric likelihood statistics based on Cressie and Read's (1984) power divergence family, which contains empirical likelihood, exponential tilting, and Pearson's $\chi^{2}$ as special cases. In this general class of nonparametric likelihood statistics, we find that certain statistics are Bartlett correctable under the constant and general non-constant volatility cases. In particular, we show that the second-order refinement of the order $O\left(n^{-2}\right)$ can be achieved for the coverage error for interval estimation or the size distortion of hypothesis testing. This Bartlett correctability can be considered as an advantage of our nonparametric likelihood approach.

In the context of high frequency data analysis, Kong (2012) has already introduced the empirical likelihood approach to conduct inference on the jump activity index by AïtSahalia and Jacod (2009b). In contrast, this paper is concerned with inference on the integrated volatility and testing for the presence of jumps, and investigates higher-order properties of the nonparametric likelihood statistics.

In addition to Gonçalves and Meddahi (2009) mentioned above, several papers have studied higher-order properties of realized volatility and related statistics in high frequency data setups to overcome poor finite sample performance of the first-order asymptotic approximations. Gonçalves and Meddahi (2008) proposed Edgeworth corrections to approximate the distribution of realized volatility. Validity of this Edgeworth expansion was established by Hounyo and Veliyev (2016). Zhang, Mykland and Aït-Sahalia (2011) studied higher-order properties of volatility related statistics under a small-noise asymptotic framework. Podolskij and Yoshida (2016) established the Edgeworth expansion for functionals of diffusion processes and applied the expansion to power variation statistics. Dovonon, Gonçalves and Meddahi (2013) considered bootstrap approximations for multivariate volatility statistics and showed that the pairs bootstrap is not second-order 
correct in general. Hounyo (2018) proposed a local Gaussian bootstrap method which can be even third-order correct. Each of these papers is concerned with approximating the distribution of realized volatility or its related statistics. In contrast, our focus is on higher-order properties of the proposed nonparametric likelihood statistics and their Bartlett correctability.

The rest of the paper is organized as follows. In Section 2, we introduce the basic idea of empirical likelihood in the context of high frequency data analysis, and discuss its general first-order asymptotic properties and some advantages compared to the existing inference methods. Section 3 applies the general first-order asymptotic theory to the baseline case (Section 3.1), and then extends it to jump robust inference (Section 3.2) and noise robust inference (Section 3.3). An empirical likelihood-based test for the presence of jumps is also proposed (Section 3.4). In Section 4, we introduce a class of nonparametric likelihood statistics, study their second-order properties, and show that some statistics are Bartlett correctable. Sections 5 and 6 present some simulation results and a real data example, respectively. Section 7 concludes. All proofs of theorems, some details for the implementation of the Bartlett correction, and additional simulation results are presented in the web appendix.

\section{EMPIRICAL LIKELIHOOD}

In this section, we introduce the basic idea of empirical likelihood in the context of high frequency data analysis (Section 2.1), present its general first-order asymptotic properties (Section 2.2), and discuss some advantages over existing inference methods (Section 2.3).

2.1. Basic idea. To fix the idea, we consider a scalar continuous time process

$$
d X_{t}=\mu_{t} d t+\sigma_{t} d W_{t}
$$


for $t \geq 0$, where $\mu$ is a drift process, $\sigma$ is a volatility process, and $W$ is a standard Brownian motion. Suppose we observe high frequency returns $r_{i}=X_{i / n}-X_{(i-1) / n}$ measured over the period $[(i-1) / n, i / n]$ for $i=1, \ldots, n$, and wish to conduct inference on a scalar functional of $\sigma$, denoted by $\theta$. A popular example is the integrated volatility $\theta=\int_{0}^{1} \sigma_{u}^{2} d u$ over $[0,1]$ (say, a day or month). For our asymptotic analysis, we consider infill asymptotics, where we take the limit $n \rightarrow \infty$ for increasingly finely sampled returns over $[0,1]$.

The basic idea for the construction of an empirical likelihood proceeds as follows. First, we take some estimating function $g\left(r_{i}, \vartheta\right)$ for the object of interest $\theta$, where the point estimator $\hat{\theta}$ solves $\sum_{i=1}^{n} g\left(r_{i}, \hat{\theta}\right)=0$. For example, the estimating function for realized volatility $\hat{\theta}=\sum_{i=1}^{n} r_{i}^{2}$ is written as $g\left(r_{i}, \vartheta\right)=n r_{i}^{2}-\vartheta$. Second, we construct an empirical likelihood at a hypothetical value $\vartheta$ based on the moment condition $E\left[g\left(r_{i}, \vartheta\right)\right]=0$. In particular, we consider the multinomial distribution with atom $w_{i}$ at point $r_{i}$ for $i=1, \ldots, n$, where its likelihood is $\prod_{i=1}^{n} w_{i}$ and the moment condition is written as $\sum_{i=1}^{n} w_{i} g\left(r_{i}, \vartheta\right)=0$. The (normalized) empirical likelihood function at $\vartheta$ is defined as

$$
E L(\vartheta)=\max _{w_{1}, \ldots, w_{n}} \prod_{i=1}^{n} n w_{i}, \quad \text { s.t. } \sum_{i=1}^{n} w_{i} g\left(r_{i}, \vartheta\right)=0, w_{i} \geq 0, \quad \sum_{i=1}^{n} w_{i}=1 .
$$

Although $E L(\vartheta)$ is a profile multinomial likelihood function, it can be used for general unknown distributions of $r_{i}$. We list some key aspects of our empirical likelihood approach.

First, although the maximization problem in (2.2) involves $n$ variables $\left(w_{1}, \ldots, w_{n}\right)$ and seems less practical, the Lagrange multiplier argument implies the convenient dual form:

$$
E L(\vartheta)=\prod_{i=1}^{n} \frac{1}{1+\lambda g\left(r_{i}, \vartheta\right)}
$$

where $\lambda$ solves $\sum_{i=1}^{n} \frac{g\left(r_{i}, \vartheta\right)}{1+\lambda g\left(r_{i}, \vartheta\right)}=0$. In practice, we employ this dual representation to evaluate $E L(\vartheta)$ since this only requires finding the root for the scalar $\lambda$. 
Second, this paper deals with interval estimation and hypothesis testing for $\theta$; we do not consider point estimation of $\theta$. Indeed, as far as the dimension of $g$ equals that of $\theta$, the maximum empirical likelihood estimator, $\arg \max _{\vartheta} E L(\vartheta)$, coincides with the conventional estimator (i.e., the solution to $\sum_{i=1}^{n} g\left(r_{i}, \hat{\theta}\right)=0$ ). We use the estimating function $g$ for interval estimation and hypothesis testing, not point estimation. In particular, we are interested in the limiting distribution of $E L(\theta)$ at the true value $\theta$, which provides a basis for testing the null $H_{0}: \theta=\vartheta$ and obtaining a confidence interval of the form $\{\vartheta: E L(\vartheta) \leq$ critical value $\}$

Finally, our formulation of the empirical likelihood is general enough to cover various objects of interest, in the form of $\theta$, and their estimating functions $g$. Furthermore, the construction of $E L(\vartheta)$ in (2.2) can naturally be extended to more general data generating processes than (2.1). These extensions will be pursued in Section 3.

2.2. General asymptotic properties. To understand the general structure of our asymptotic analysis for $E L(\theta)$, we first present a general result to characterize the first-order asymptotic properties of $E L(\theta)$ under some high level conditions. Let $\bar{g}=n^{-1} \sum_{i=1}^{n} g\left(r_{i}, \theta\right)$ and $\bar{V}=n^{-1} \sum_{i=1}^{n} g\left(r_{i}, \theta\right)^{2}$, where both $g$ and $\theta$ are scalar. The following theorem is an adapted version of Hjort, McKeague and van Keilegom (2009, Theorem 2.1).

Theorem 1. Suppose (i) $\sqrt{\frac{n}{V_{g}}} \bar{g} \stackrel{d}{\rightarrow} N(0,1)$ for some $V_{g}>0$, (ii) $\bar{V} \stackrel{p}{\rightarrow} V$ for some $V>0$, (iii) the probability that the origin is contained in the interior of the convex hull of $\left\{g\left(r_{i}, \theta\right)\right\}_{i=1}^{n}$ converges to zero, and (iv) $\max _{1 \leq i \leq n}\left|g\left(r_{i}, \theta\right)\right|=o_{p}(\sqrt{n})$. Then for any $\hat{A} \stackrel{p}{\rightarrow} V / V_{g}$,

$$
T_{E L}(\theta)=\hat{A}\{-2 \log E L(\theta)\} \stackrel{d}{\rightarrow} \chi_{1}^{2} .
$$

This theorem says that the (modified) empirical likelihood statistic $T_{E L}(\theta)$ is asymptotically pivotal and converges to the $\chi_{1}^{2}$ distribution under the high level conditions (i)-(iii). 
In the next section, we apply this general theorem to specific moment functions $g$ and data generating processes of $\left\{r_{i}\right\}$. Based on this theorem, the $100(1-\alpha) \%$ empirical likelihood confidence set for $\theta$ is given by $C I_{E L}^{\alpha}=\left\{\vartheta: T_{E L}(\vartheta) \leq \chi_{1, \alpha}^{2}\right\}$, where $\chi_{1, \alpha}^{2}$ is the $(1-\alpha)$-th quantile of the $\chi_{1}^{2}$ distribution. Furthermore, hypothesis tests on $\theta$ can be implemented using the $\chi_{1}^{2}$ distribution.

A major difference in the asymptotic result of (2.4) from the conventional one for i.i.d. observations is the presence of the adjustment term $\hat{A}$ which facilitates the convergence to the chi-squared distribution. If the sample $\left\{r_{i}\right\}_{i=1}^{n}$ is i.i.d., then it holds that $V_{g}=V$ and (2.4) is satisfied with $\hat{A}=1$. On the other hand, under infill asymptotics, $V_{g}$ and $V$ typically do not coincide, and the adjustment term $\hat{A}$ in $(2.4)$ is required to recover asymptotic pivotalness. The next section provides several examples of $\hat{A}$.

Conditions (i) and (ii) are key to obtain the chi-squared limiting distribution in (2.4), and can be verified by applying certain central limit theorems and laws of large numbers, respectively. Condition (iii) is required to guarantee the existence of the solution in (2.2). Condition (iv) is a mild regularity condition to establish a quadratic approximation for the object $-2 \log E L(\theta)$.

The proof of this theorem can be found in p. 1105 of Hjort, McKeague and van Keilegom (2009). The basic steps are: first establish the quadratic approximation $-2 \log E L(\theta)=$ $\bar{V}^{-1}(\sqrt{n} \bar{g})^{2}+o_{p}(1)$, and then apply the continuous mapping theorem by Conditions (i)-(ii).

2.3. Advantages of empirical likelihood-based inference. In this section, we discuss several advantages of empirical likelihood-based inference. In particular, we compare the empirical likelihood confidence interval $C I_{E L}^{\alpha}$ defined in the last subsection with the conventional Wald-type confidence interval $C I_{W}^{\alpha}=\left[\hat{\theta} \pm z_{\alpha / 2} \cdot\right.$ standard error $]$, where $z_{\alpha / 2}$ 
is the $(1-\alpha / 2)$-th quantile of the standard normal distribution. Our discussion here is based on Hall and LaScala (1990).

First, $C I_{E L}^{\alpha}$ is not shaped in a predetermined way and may be asymmetric around the point estimate $\hat{\theta}$. The symmetric shape constraint in $C I_{W}^{\alpha}$ imposes a degree of nonexistent symmetry in the sampling distribution.

Second, $C I_{E L}^{\alpha}$ tends to be concentrated in a region where the density of $\hat{\theta}$ is high. In other words, the shape of $C I_{E L}^{\alpha}$ automatically reflects the emphasis in the observed data. Third, related to the above points, $C I_{E L}^{\alpha}$ naturally satisfies restrictions on the range of $\theta$. For example, if $\theta$ is the integrated volatility, $C I_{E L}^{\alpha}$ never contains negative values, whereas the lower endpoint of $C I_{W}^{\alpha}$ may be negative. This point will be illustrated in our simulation study. ${ }^{1}$

Fourth, $C I_{E L}^{\alpha}$ is transformation respecting (i.e., the confidence interval of $f(\theta)$ is given by $\left\{f(\theta): \theta \in C I_{E L}^{\alpha}\right\}$. However, $C I_{W}^{\alpha}$ is not invariant to transformations of $\theta$ and may yield different conclusions.

In addition to these points, there is an important potential advantage of empirical likelihood: Bartlett correctability. The Bartlett correction, an analytical higher-order refinement, is an adjustment of the mean of the empirical likelihood statistic (to be closer to the $\chi_{1}^{2}$ distribution) so that the adjusted confidence interval has better coverage accuracy. Although Bartlett correctability of empirical likelihood is reported in various contexts (see, Chapter 13 of Owen, 2001), it is an open question whether a similar phenomenon emerges in high frequency data setups.

Our formal analysis of Bartlett correctability will be presented in Section 4. Here we provide some background for our higher-order analysis based on Hall and LaScala

\footnotetext{
${ }^{1}$ To avoid the negative lower endpoint, several papers considered the Wald confidence interval based on the logarithmic transformed object, $\log \theta$ (e.g., Barndorff-Nielsen and Shephard, 2005, and Gonçalves and Meddahi, 2009 and 2011). See Section 5.1 for some simulation results on this approach.
} 
(1990). Intuitively, a part of the approximation error in (2.4) is due to a discrepancy in the mean of the statistic (i.e., $E\left[T_{E L}(\theta)\right] \neq 1$ ). This discrepancy can be eliminated by the ratio $T_{E L}(\theta) / E\left[T_{E L}(\theta)\right]$. Thus the Bartlett correction for $T_{E L}(\theta)$ takes the form of $T_{E L}(\theta) /\left(1+n^{-1} a\right)$, where $E\left[T_{E L}(\theta)\right]=1+n^{-1} a+O\left(n^{-2}\right)$.

Let $S$ be the asymptotic signed root of $T_{E L}(\theta)$ satisfying $T_{E L}(\theta)=S^{2}+O_{p}\left(n^{-3 / 2}\right)$

and $S \stackrel{d}{\rightarrow} N(0,1)$. To induce higher-order refinement of the corrected confidence interval $\left\{\vartheta: T_{E L}(\vartheta) /\left(1+n^{-1} a\right) \leq \chi_{1, \alpha}^{2}\right\}$, a key condition is that the third and fourth order cumulants of $S$ are close enough to those of the standard normal distribution and of order $O\left(n^{-3}\right)$ and $O\left(n^{-4}\right)$, respectively (see, pp. 116-119 of Hall and LaScala, 1990, for detail).

A major issue of applying this general result to our setup is that the third and fourth order cumulants of the signed root term $S$ may not vanish at the above rates under infill asymptotics. Note that $S$ depends on various empirical moments of $r_{i}$ which show rather different limiting behaviors under infill asymptotics compared to the case of i.i.d. observations. Therefore, the empirical likelihood statistic $T_{E L}(\theta)$ may not be Bartlett correctable. We analyze this problem in Section 4 by considering a general class of nonparametric likelihood statistics, where some statistics in this class can be Bartlett correctable.

\section{First-ORDER ASYMPTOTIC THEORY}

In this section, we apply the general first-order asymptotic theory for the empirical likelihood statistic in Theorem 1 to a benchmark setup (Section 3.1), and then extend the result to jump robust inference (Section 3.2) and noise robust inference (Section 3.3). In Section 3.4, we modify our approach to test for the presence of jumps.

3.1. Benchmark case. In this subsection, we consider the benchmark setup in (2.1) and impose the following assumption based on Barndorff-Nielsen et al. (2006). 
Assumption X. The process $X$ defined on a filtered probability space follows (2.1), where $\mu$ is an adapted predictable locally bounded drift process, and $\sigma$ is an almost surely positive and adapted cadlag volatility process satisfying

$$
\sigma_{t}=\sigma_{0}+\int_{0}^{t} a_{u}^{*} d u+\int_{0}^{t} \sigma_{u-}^{*} d W_{u}+\int_{0}^{t} v_{u-}^{*} d V_{u}
$$

where $a^{*}, \sigma^{*}$, and $v^{*}$ are adapted cadlag processes, $a^{*}$ is a predictable and locally bounded process, and $V$ is a Brownian motion independent of $W$ in (2.1).

This assumption is general enough to allow for intraday seasonality and correlation between $\sigma$ and $W$ (called the leverage effect).

We first consider inference on the integrated volatility $\theta=\int_{0}^{1} \sigma_{u}^{2} d u$. One popular estimator of $\theta$ is the realized volatility statistic $\hat{\theta}=\sum_{i=1}^{n} r_{i}^{2}$. Under Assumption $\mathrm{X}, \hat{\theta}$ is consistent and asymptotically normal, $\hat{V}^{-1 / 2} \sqrt{n}(\hat{\theta}-\theta) \stackrel{d}{\rightarrow} N(0,1)$ as $n \rightarrow \infty$, where $\hat{V}=\frac{2 n}{3} \sum_{i=1}^{n} r_{i}^{4}$ (Barndorff-Nielsen et al., 2006). Based on this result, it is customary to construct a Wald-type confidence interval for $\theta$.

Based on the estimating equation $\sum_{i=1}^{n}\left(n r_{i}^{2}-\hat{\theta}\right)=0$ for the realized volatility $\hat{\theta}$, the empirical likelihood function $E L(\vartheta)$ can be defined as in $(2.2)$ with $g\left(r_{i}, \vartheta\right)=n r_{i}^{2}-\vartheta$. Let $R_{q}=n^{q / 2-1} \sum_{i=1}^{n}\left|r_{i}\right|^{q}$. By applying Theorem 1 to this setup, the first-order asymptotic distribution of the empirical likelihood statistic is obtained as follows.

Theorem 2. Under Assumption $X, T_{E L}(\theta)$ with $g\left(r_{i}, \theta\right)=n r_{i}^{2}-\theta$ satisfies (2.4) for $\hat{A}=\frac{3}{2}\left(1-\frac{R_{2}^{2}}{R_{4}}\right)$.

This theorem is shown by verifying Conditions (i)-(iv) in Theorem 1. The proof is presented in the web appendix. Based on this theorem, the empirical likelihood confidence interval is given by $C I_{E L}^{\alpha}=\left\{\vartheta: T_{E L}(\vartheta) \leq \chi_{1, \alpha}^{2}\right\}$. See Section 2.3 for advantages of $C I_{E L}^{\alpha}$. 
We next consider the $p$-th power variation $\theta_{p}=\int_{0}^{1} \sigma_{u}^{p} d u$ for $p>0$. By Barndorff-Nielsen et al. (2006), $\theta_{p}$ is consistently estimated by $\hat{\theta}_{p}=\mu_{p}^{-1} n^{-1+p / 2} \sum_{i=1}^{n}\left|r_{i}\right|^{p}$, where $\mu_{p}=E|z|^{p}$ with $z \sim N(0,1)$. Based on the estimating equation $\sum_{i=1}^{n}\left(\mu_{p}^{-1} n^{p / 2}\left|r_{i}\right|^{p}-\hat{\theta}_{p}\right)=0$ for $\hat{\theta}_{p}$, the empirical likelihood function for $\theta_{p}$ can be constructed as in $(2.2)$ with $g\left(r_{i}, \vartheta_{p}\right)=$ $\mu_{p}^{-1} n^{p / 2}\left|r_{i}\right|^{p}-\vartheta_{p}$ and the first-order asymptotic distribution of the empirical likelihood statistic is obtained as follows.

Theorem 3. Under Assumption $X, T_{E L}\left(\theta_{p}\right)$ with $g\left(r_{i}, \theta_{p}\right)=\mu_{p}^{-1} n^{p / 2}\left|r_{i}\right|^{p}-\theta_{p}$ satisfies (2.4) for $\hat{A}=\frac{\mu_{2 p} R_{2 p}-\mu_{p}^{2} R_{p}}{\left(\mu_{2 p}-\mu_{p}^{2}\right) R_{2 p}}$.

Similar comments to Theorem 3.1 apply. As we explained in Section 2.1, the above theorems provide new interval estimation and hypothesis testing methods for $\theta$ and $\theta_{p}$, not new point estimators. Indeed the maximizers of $T_{E L}(\theta)$ and $T_{E L}\left(\theta_{p}\right)$ coincide with the conventional estimators, $\hat{\theta}$ and $\hat{\theta}_{p}$, respectively. The issue of optimal testing (or interval estimation) for $\theta$ or $\theta_{p}$ would be an interesting avenue for future research. In a recent paper, Renault, Sarisoy and Werker (2017) studied efficient point estimation of $\theta, \theta_{p}$, and related objects, and discussed efficient properties of the existing estimators, such as the ones by Mykland and Zhang (2009) and Jacod and Rosenbaum (2013). We conjecture that the empirical likelihood-based tests using the estimating equations of these efficient estimators will enjoy some optimal local power properties. Formal analysis of this issue requires developing the notion and theory of semiparametric efficient testing in the context of high frequency data analysis (cf. Choi, Hall and Schick, 1996, for the case of i.i.d. observations) and is, thus, beyond the scope of this paper.

3.2. Jump robust inference. In this subsection, we propose a jump robust version of the empirical likelihood statistic for the integrated volatility $\theta$. The empirical likelihood 
statistic in Theorem 2 is constructed from the estimating equation for the realized volatility $\hat{\theta}=\sum_{i=1}^{n} r_{i}^{2}$. Our approach can be generalized to other estimating equations for the integrated volatility $\theta$. In particular, we consider the multipower variation (e.g., BarndorffNielsen and Shephard, 2004, and Barndorff-Nielsen, Shephard and Winkel, 2006)

$$
\hat{\theta}_{\mathbf{p}}=\sum_{i=m}^{n}\left|r_{i-m+1}\right|^{p_{1}} \cdots\left|r_{i}\right|^{p_{m}}
$$

for a vector $\mathbf{p}=\left(p_{1}, \ldots, p_{m}\right)$ of positive numbers satisfying $p_{1}+\cdots+p_{m}=2$. Indeed, the realized volatility is a special case of $\hat{\theta}_{\mathbf{p}}$ (with $m=1$ and $p_{1}=2$ ). A remarkable property of the multipower variation is: if $p$ 's are reasonably small (see (3.3) below), then the estimator $\hat{\theta}_{\mathbf{p}}$ enjoys certain robustness against jumps in the observed process.

To be precise, consider the process

$$
Y_{t}=X_{t}+J_{t}
$$

for $t \geq 0$, where $X$ is generated by the continuous time process in (2.1) satisfying Assumption $\mathrm{X}$, and $J$ is a jump process, which is assumed to be a Lévy process with no continuous component and index $\alpha=\inf \left\{a \geq 0: \int_{[-1,1]}|x|^{a} \Pi(d x)<\infty\right\} \in[0,2]$ for the Lévy measure П. The Lévy process is a convenient general class of processes to accommodate both finite and infinite activity jumps. Barndorff-Nielsen, Shephard and Winkel (2006, Theorem 1) showed that the limiting distribution of the multipower variation $\hat{\theta}_{\mathbf{p}}$ remains the same regardless of the presence of the jump process $J$ so long as

$$
\alpha<1, \quad \frac{\alpha}{2-\alpha} \leq \min \left\{p_{1}, \ldots, p_{m}\right\} \leq \max \left\{p_{1}, \ldots, p_{m}\right\}<1
$$

A popular choice of $\mathbf{p}$ for the jump robust estimator is the tripower variation (i.e., $m=3$ and $\left.p_{1}=p_{2}=p_{3}=2 / 3\right)$. 
Suppose we observe high frequency returns $\tilde{r}_{i}=Y_{i / n}-Y_{(i-1) / n}$ measured over the period $[(i-1) / n, i / n]$ for $i=1, \ldots, n$. Let $c_{\mathbf{p}}=\prod_{l=1}^{m} \mu_{p_{l}}$, where $\mu_{p}=E|z|^{p}$ with $z \sim N(0,1)$. Based on the estimating equation for $\hat{\theta}_{\mathbf{p}}$, we define the jump robust empirical likelihood function for $\theta$ as in $(2.2)$ with $g_{i}(\vartheta)=n\left|\tilde{r}_{i-m+1}\right|^{p_{1}} \cdots\left|\tilde{r}_{i}\right|^{p_{m}}-c_{\mathbf{p}} \vartheta$. Let $\tilde{R}_{2}=\hat{\theta}_{\mathbf{p}}$ and $\tilde{R}_{4}=n \sum_{i=m}^{n}\left|\tilde{r}_{i-m+1}\right|^{2 p_{1}} \cdots\left|\tilde{r}_{i}\right|^{2 p_{m}}$. Define the constant

$$
d_{\mathbf{p}}=\prod_{l=1}^{m} \mu_{2 p_{l}}-(2 m-1) \prod_{l=1}^{m} \mu_{p_{l}}^{2}+2 \sum_{k=1}^{m-1} \prod_{l=1}^{k} \mu_{p_{k}} \prod_{l=m-k+1}^{m} \mu_{p_{l}} \prod_{l=1}^{m-k} \mu_{p_{l}+p_{l+k}} .
$$

Then the first-order asymptotic properties of the jump robust empirical likelihood statistic are obtained as follows.

Theorem 4. Suppose $Y$ is generated by (3.2). Assume $p_{1}+\cdots+p_{m}=2$ and (3.3). Then $T_{E L}(\theta)$ with $g_{i}(\theta)=n\left|\tilde{r}_{i-m+1}\right|^{p_{1}} \cdots\left|\tilde{r}_{i}\right|^{p_{m}}-c_{\mathbf{p}} \theta$ satisfies (2.4) for $\hat{A}=\frac{c_{2 \mathbf{p}}}{d_{\mathbf{p}}}\left(1-\frac{\tilde{R}_{2}^{2}}{\tilde{R}_{4}}\right)$. This result does not change even if $J=0$ (the case of no jump).

This theorem says that the empirical likelihood statistic $T_{E L}(\theta)$ using the above $g$ has the limiting $\chi_{1}^{2}$ distribution which is invariant to the presence of jumps. The jump robust confidence interval for $\theta$ is obtained in the same manner. We note that the empirical likelihood function for the benchmark case (i.e., $m=1$ and $p_{1}=2$ ) does not satisfy the condition in (3.3).

3.3. Noise robust inference. Our empirical likelihood approach can be adapted to be robust to the presence of microstructure noise. In particular, we employ the pre-averaging approach of Jacod et al. (2009), and construct an empirical likelihood based on block averages of the original data. In this subsection, we consider the following setup. 
Assumption X'. Observations $\left\{Z_{i / n}\right\}_{i=1}^{n}$ are generated from $Z_{i / n}=X_{i / n}+U_{i / n}$, where $\left\{X_{i / n}\right\}_{i=1}^{n}$ is drawn from the latent process $X$ satisfying Assumption $X$, and $\left\{U_{i / n}\right\}_{i=1}^{n}$ is an i.i.d. sequence with zero mean and finite eighth moments and is independent of $X$.

We are interested in the integrated volatility $\theta=\int_{0}^{1} \sigma_{u}^{2} d u$ of the latent process $X$. It is known that due to the presence of the noise term $U_{i / n}$, the conventional realized volatility based on the observables $\left\{Z_{i / n}\right\}_{i=1}^{n}$ is inconsistent for $\theta$ (e.g., Hansen and Lunde, 2006, and Bandi and Russell, 2008).

In this setup, Jacod et al. (2009) developed a noise robust estimator for $\theta$ based on the pre-averaging approach. A simplified version of their estimator is described as follows. First, we transform the observed data $\left\{Z_{i / n}\right\}_{i=1}^{n}$ into block averages $\bar{Z}_{i / n}=$ $K^{-1} \sum_{j=0}^{K-1} Z_{(i+j) / n}$ for $i=0,1, \ldots, n-K+1$. Second, based on the block averages, compute (half of) the return data $\bar{r}_{i}=\left(\bar{Z}_{(i+K) / n}-\bar{Z}_{i / n}\right) / 2$ for $i=1, \ldots, n-K+1$. Finally, compute the noise robust estimator as

$$
\bar{\theta}=\frac{6}{K} \sum_{i=1}^{n_{K}} \bar{r}_{i}^{2}-\frac{3}{2 K^{2}} \hat{\theta},
$$

where $n_{K}=n-2 K+2$ and $\hat{\theta}=\sum_{i=1}^{n}\left(Z_{i / n}-Z_{(i-1) / n}\right)^{2}$ is the conventional realized volatility estimator using the original data. Intuitively, compared to the original $Z_{i / n}$, the variance of the noise in the block average $\bar{Z}_{i / n}$ is reduced by a factor of $1 / K$. Thus, the volatility estimator $\bar{\theta}$ based on the block averages is expected to be less sensitive to the presence of the noise term. The second term in (3.4) is a bias correction term. Jacod et al. (2009) showed $\bar{\theta}$ is consistent for $\theta$ and asymptotically normal with the rate $n^{-1 / 4}$.

Using the estimating equation for (3.4), we define the noise robust empirical likelihood function for $\theta$ as in (2.2) with $g_{i}(\vartheta)=\frac{6 n_{K}}{K} \bar{r}_{i}^{2}-\frac{3}{2 K^{2}} \hat{\theta}-\vartheta$ for $i=1, \ldots, n_{K}$. Choose the 
block length as $K=\frac{1}{2} c n^{1 / 2}+o\left(n^{1 / 4}\right)$ for some $c>0$. Then define $\bar{R}_{q}=n_{K}^{q / 2-1} \sum_{i=1}^{n_{K}}\left|\bar{r}_{i}\right|^{q}$,

$$
\begin{aligned}
R_{4}^{*}= & \frac{4 \cdot 12^{4} \Phi_{22}}{3 c} \sum_{i=1}^{n_{K}} \bar{r}_{i}^{4}+\frac{4}{c^{3} n}\left(12^{3} \Phi_{12}-12^{4} \Phi_{22}\right) \sum_{i=1}^{n_{K}-2 K} \bar{r}_{i}^{2} \sum_{j=i+2 K}^{i+4 K-1}\left(Z_{j / n}-Z_{(j-1) / n}\right)^{2} \\
& +\frac{1}{c^{3} n}\left(12^{2} \Phi_{11}-2 \cdot 12^{3} \Phi_{12}+12^{4} \Phi_{22}\right) \sum_{i=2}^{n-2}\left(Z_{i / n}-Z_{(i-1) / n}\right)^{2}\left(Z_{(i+2) / n}-Z_{(i+1) / n}\right)^{2}
\end{aligned}
$$

with $\Phi_{11}=\frac{1}{6}, \Phi_{12}=\frac{1}{96}$, and $\Phi_{22}=\frac{151}{80640}$. The object $R_{4}^{*}$ appeared in Jacod et al. (2009, eq. (3.7)) as an estimator of the asymptotic variance of $\bar{\theta}$. The first-order asymptotic distribution of the noise robust empirical likelihood statistic is obtained as follows.

Theorem 5. Under Assumption $X^{\prime}, T_{E L}(\theta)$ with $g_{i}(\theta)=\frac{6 n_{K}}{K} \bar{r}_{i}^{2}-\frac{3}{2 K^{2}} \hat{\theta}-\theta$ satisfies (2.4) for $\hat{A}=\frac{36 n^{1 / 2}}{n_{K} K^{2}} \frac{\bar{R}_{4}-\bar{R}_{2}^{2}}{R_{4}^{*}}$.

As pointed out by Jacod et al. (2009), the pre-averaging estimator $\bar{\theta}$ can be interpreted as a realized kernel estimator in Barndorff-Nielsen et al. (2008). Similarly, our empirical likelihood statistic $T_{E L}(\theta)$ using the block averages may be interpreted as the block empirical likelihood statistic by Kitamura (1997) for weakly dependent data. However, the block averages here are employed to reduce the effect of microstructure noise.

In this section, we impose Assumption $\mathrm{X}^{\prime}$ and consider the case of additive and i.i.d. noise for simplicity. We conjecture that it is possible to extend our approach to more general setups, such as weakly dependent noise (Aït-Sahalia, Mykland and Zhang, 2011), non-additive noise (Jacod et al., 2009), and endogenous time (Li, Zhang and Zheng, 2013) by modifying the estimating function to be robust to those setups.

3.4. Test for presence of jumps. So far, we have considered empirical likelihood inference on some functional $\theta$ of the volatility process $\sigma$. However, the empirical likelihood approach can be used for other purposes. An important application is to test whether different estimating functions for the same object converge to the same limit. 
For example, consider the setup in Section 3.2 and suppose we wish to test the presence of jumps in the observed process (i.e., $J$ in $(3.2)$ ). When there is no jump in the process (i.e., $J=0$ ), both the multipower variation $\hat{\theta}_{\mathbf{p}}$ in $(3.1)$ (with $p_{1}+\cdots+p_{m}=2$ ) and the realized volatility $c_{\mathbf{p}} \sum_{i=1}^{n} \tilde{r}_{i}^{2}$ multiplied by the constant $c_{\mathbf{p}}$ are consistent for $c_{\mathbf{p}} \theta$. Therefore, the object $\sum_{i=m}^{n}\left(\left|\tilde{r}_{i-m+1}\right|^{p_{1}} \cdots\left|\tilde{r}_{i}\right|^{p_{m}}-c_{\mathbf{p}} \tilde{r}_{i}^{2}\right)$ converges to zero and the empirical likelihood statistic tends to be small. On the other hand, in the presence of jumps, this object converges to a negative constant (Barndorff-Nielsen and Shephard, 2004) and the empirical likelihood statistic tends to be large.

Let $c_{\tilde{\mathbf{p}}_{l}}=\left(\mu_{p_{l}+2} / \mu_{p_{l}}\right) \prod_{k=1}^{m} \mu_{p_{k}}$ be a known constant. The first-order asymptotic properties of the empirical likelihood statistic for the presence of jumps are obtained as follows.

Theorem 6. Suppose $Y=X$ (i.e., no jump), where $X$ satisfies Assumption $X$. Then $T_{E L}$ with $g_{i}=\left|\tilde{r}_{i-m+1}\right|^{p_{1}} \cdots\left|\tilde{r}_{i}\right|^{p_{m}}-c_{\mathbf{p}} \tilde{r}_{i}^{2}$ satisfies (2.4) for $\hat{A}=\frac{c_{2 \mathbf{p}}+3 c_{\mathbf{p}}^{2}-2 c_{\mathbf{p}} c_{\tilde{\mathbf{p}}}}{d_{\mathbf{p}}-2 \sum_{l=1}^{m} c_{\mathbf{p}}\left(c_{\tilde{\mathbf{p}}_{l}}-c_{\mathbf{p}}\right)+2 c_{\mathbf{p}}^{2}}$. On the other hand, if $Y$ is generated by (3.2), then the statistic $T_{E L}$ diverges.

Since $\sum_{i=m}^{n} g_{i}$ converges to a negative constant under the alternative hypothesis, we propose a one-sided version of the (signed root) empirical likelihood statistic $S_{E L}=$ $-\operatorname{sgn}\left(\sum_{i=m}^{n} g_{i}\right) T_{E L}^{1 / 2}$. Based on the above theorem, we reject the null of no jump if $S_{E L}>z_{1-\alpha}$ where $z_{1-\alpha}$ is the $(1-\alpha)$-th quantile of the standard normal distribution.

Note that the adjustment term $\hat{A}$ is a known constant in this case. Among several existing methods for testing the presence of jumps (see, Dumitru and Urga, 2012, for a review), our empirical likelihood test can be considered as a likelihood ratio counterpart of the Wald-type tests by Barndorff-Nielsen and Shephard (2004) and Huang and Tauchen (2005) (for the special case of $\mathbf{p}=(1,1)$ ) which essentially tests whether the ratio of the bipower variation to the realized volatility (i.e., $\left.\hat{\theta}_{(1,1)} / \hat{\theta}_{2}\right)$ is different from one. 
Based on the discussion in Section 2.3, advantages of our empirical likelihood jump test compared to the Wald-type tests are as follows. First, the empirical likelihood statistic $T_{E L}$ does not require estimation of the scale for any $\mathbf{p}$ (again, $\hat{A}$ in Theorem 6 is a known constant). However, the Wald statistic based on $\hat{\theta}_{\mathbf{p}} / \hat{\theta}_{2}$ requires estimation of the standard error for each $\mathbf{p}$, and, generally, scale estimation of higher moments is a difficult task. Second, the Wald-type tests are not invariant to formulations of the test statistics. For

example, the Wald statistics $\frac{\hat{\theta}_{(1,1)} / \hat{\theta}_{2}-1}{\left.\text { s.e. } \hat{\theta}_{(1,1)} / \hat{\theta}_{2}\right)}$ and $\frac{\hat{\theta}_{(1,1)}-\hat{\theta}_{2}}{\text { s.e. }\left(\hat{\theta}_{(1,1)}-\hat{\theta}_{2}\right)}$ generally yield different outcomes and the conclusion can be different. On the other hand, the empirical likelihood statistic is free from such lack of invariance. Third, in the literature on empirical likelihood, the above attractive features are typically coupled by better higher-order properties (in particular, Bartlett correctability). Although it is beyond the scope of this paper, we conjecture that the generalized likelihood version of $T_{E L}$ as in Section 4 would provide a Bartlett correctable statistic.

\section{General nonparametric likelihood And SECOnd-Order ASymptotics}

In this section, we generalize the construction of nonparametric likelihood for the integrated volatility by using the power divergence family (Cressie and Read, 1984). This family is general enough to accommodate not only the empirical likelihood considered so far, but also other likelihood functions. Based on this general family of nonparametric likelihood functions, we investigate second-order asymptotic properties of nonparametric likelihood statistics. In particular, we show that adequate choices of tuning constants lead to Bartlett correctable statistics.

4.1. General nonparametric likelihood. We first consider the benchmark setup in Section 3.1. As a general family of nonparametric likelihood functions, we employ the 
power divergence family (Cressie and Read, 1984)

$$
L_{\gamma}\left(w_{1} \ldots, w_{n}\right)=\frac{2}{\gamma(\gamma+1)} \sum_{i=1}^{n}\left\{\left(n w_{i}\right)^{\gamma+1}-1\right\}
$$

for $\gamma \neq-1,0$. For $\gamma=-1$ and $0, L_{\gamma}\left(w_{1} \ldots, w_{n}\right)=-2 \sum_{i=1}^{n} \log \left(n w_{i}\right)$ and $2 n \sum_{i=1}^{n} w_{i} \log \left(n w_{i}\right)$, respectively. Based on $L_{\gamma}\left(w_{1} \ldots, w_{n}\right)$ and using the estimating equation for the realized volatility $\hat{\theta}=\sum_{i=1}^{n} r_{i}^{2}$, we specify the likelihood function for the integrated volatility $\theta$ as $\ell_{\gamma, \phi}(\theta)=L_{\gamma}\left(w_{\phi, 1} \ldots, w_{\phi, n}\right)$, where the weights $w_{\phi, 1}, \ldots, w_{\phi, n}$ solve

$$
\min _{w_{1}, \ldots, w_{n}} L_{\phi}\left(w_{1} \ldots, w_{n}\right), \quad \text { subject to } \sum_{i=1}^{n} w_{i}=1, \quad \sum_{i=1}^{n} w_{i}\left(n r_{i}^{2}-\theta\right)=0 \text {. }
$$

The nonparametric likelihood function $\ell_{\gamma, \phi}(\theta)$ contains two tuning constants, $\gamma$ and $\phi$. In the literature, it is commonly assumed $\gamma=\phi$. For example, the empirical likelihood function corresponds to $\gamma=\phi=-1$, and Pearson's $\chi^{2}$ corresponds to $\gamma=\phi=-2$.

Baggerly (1998) showed that in the class of likelihood functions with $\gamma=\phi$, only empirical likelihood is Bartlett correctable for the mean of i.i.d. data. On the other hand, Schennach $(2005,2007)$ considered the case of $\gamma \neq \phi$ and studied the exponentially tilted empirical likelihood statistic with $\gamma=-1$ and $\phi=0$ from both a Bayesian and a frequentist perspective. In our infill asymptotics setup, it is crucial to consider the general class of $\ell_{\gamma, \phi}(\theta)$ indexed by $\gamma$ and $\phi$ to achieve a Bartlett correction. Below, we will show that even if the volatility process $\sigma$ is constant, the empirical likelihood statistic (i.e., $\ell_{\gamma, \phi}(\theta)$ with $\gamma=\phi=-1$ ) is not Bartlett correctable under infill asymptotics, and the constants $\gamma$ and $\phi$ need to be chosen separately to achieve Bartlett correction. This is because under infill asymptotics the empirical moments contained in an expansion of $\ell_{\gamma, \phi}(\theta)$ follow rather different laws of large numbers compared to the i.i.d. case. 
By the Lagrange multiplier argument, the solution of (4.1) is (see, Baggerly, 1998)

$$
w_{\phi, i}=\frac{1}{n}\left(1+\eta+\lambda\left(n r_{i}^{2}-\theta\right)\right)^{\frac{1}{\phi}},
$$

for $\phi \neq 0$ and $w_{\phi, i}=\frac{1}{n} \eta \exp \left(\lambda\left(n r_{i}^{2}-\theta\right)\right)$ for $\phi=0$, where $\eta$ and $\lambda$ solve

$$
\frac{1}{n} \sum_{i=1}^{n}\left(1+\eta+\lambda\left(n r_{i}^{2}-\theta\right)\right)^{\frac{1}{\phi}}=1, \quad \frac{1}{n} \sum_{i=1}^{n}\left(1+\eta+\lambda\left(n r_{i}^{2}-\theta\right)\right)^{\frac{1}{\phi}}\left(n r_{i}^{2}-\theta\right)=0
$$

for $\phi \neq 0$ and solve $\frac{1}{n} \sum_{i=1}^{n} \eta \exp \left(\lambda\left(n r_{i}^{2}-\theta\right)\right)=1$ and $\frac{1}{n} \sum_{i=1}^{n} \eta \exp \left(\lambda\left(n r_{i}^{2}-\theta\right)\right)\left(n r_{i}^{2}-\theta\right)=0$ for $\phi=0$. In practice, we use (4.2) to compute the weights in (4.1).

The first-order asymptotic distribution of $\ell_{\gamma, \phi}(\theta)$ is obtained as follows.

Theorem 7. Suppose Assumption $X$ holds true. For each $\gamma, \phi \in \mathbb{R}$, as $n \rightarrow \infty$,

$$
T_{\gamma, \phi}(\theta)=\frac{3}{2}\left(1-\frac{R_{2}^{2}}{R_{4}}\right) \ell_{\gamma, \phi}(\theta) \stackrel{d}{\rightarrow} \chi_{1}^{2}
$$

Similar comments to Theorem 2 apply. Similar modifications as in Sections 3.2 and 3.3 can be applied to $T_{\gamma, \phi}(\theta)$ to be robust to jumps and microstructure noise.

Note that the first-order asymptotic distribution of the statistic $T_{\gamma, \phi}(\theta)$ is identical to the one in Theorem 2 for the empirical likelihood. Moreover, the first-order asymptotic distribution does not depend on the tuning constants $\gamma$ and $\phi$. The next subsection studies second-order asymptotic properties of $T_{\gamma, \phi}(\theta)$ to compare different choices of $\gamma$ and $\phi$.

4.2. Second-order asymptotics. The first-order asymptotic theory for $T_{\gamma, \phi}(\theta)$ in Theorem 7 is silent about the choice of the tuning constants $\gamma$ and $\phi$. In order to address this issue, we investigate the second-order asymptotic properties of $T_{\gamma, \phi}(\theta)$. Following the conventional recipe described in Section 2.3 (see also, DiCiccio, Hall and Romano, 1991, 
and Baggerly, 1998, among others), we first derive the signed root $S_{\gamma, \phi}$ of the nonparametric likelihood statistic satisfying $T_{\gamma, \phi}(\theta)=S_{\gamma, \phi}+O_{p}\left(n^{-3 / 2}\right)$, then evaluate the cumulants of the signed root. In particular, based on the third and fourth cumulants of $S_{\gamma, \phi}$ (say, $\kappa_{\gamma, \phi}^{(3)}$ and $\left.\kappa_{\gamma, \phi}^{(4)}\right)$, we seek values of $\gamma$ and $\phi$ at which $\kappa_{\gamma, \phi}^{(3)}$ and $\kappa_{\gamma, \phi}^{(4)}$ vanish at sufficiently fast rates to achieve a Bartlett correction. Details are provided in the web appendix (proofs of Theorems 8 and 9). For the second-order analysis, we add the following assumption.

Assumption H. The process $X$ follows (2.1) with $\mu=0$ and $\sigma$ is independent of $W$ and bounded away from zero.

This assumption is restrictive since it rules out the drift term and leverage effect. Gonçalves and Meddahi (2009, p. 289) imposed a similar but stronger assumption for their higher-order analysis of bootstrap inference. Although the drift term $\mu$ is asymptotically negligible of the first-order, it will appear in the higher-order terms and complicates our second-order analysis. Ruling out the leverage effect (i.e., independence between $\sigma$ and $W)$ also simplifies our second-order analysis since it allows us to condition on the path of $\sigma$ to compute the cumulants of the signed root $S_{\gamma, \phi}$. Relaxing Assumption $\mathrm{H}$ for the second-order analysis is beyond the scope of this paper.

To simplify the exposition of our results, we first consider the simple case where the volatility is constant $\left(\sigma_{t}=\sigma\right.$ over $\left.t \in[0,1]\right)$. In this setting, the second-order properties of the nonparametric likelihood statistic $T_{\gamma, \phi}(\theta)$ are presented as follows.

Theorem 8. Suppose Assumptions $X$ and $H$ hold true and $\sigma_{t}=\sigma$ over $t \in[0,1]$. Then, for $\gamma=-1$ and $\phi=-1 \pm \frac{\sqrt{5}}{3}$, the nonparametric likelihood statistic $T_{\gamma, \phi}(\theta)$ is Bartlett correctable, i.e., conditionally on the path of $\sigma$,

$$
\operatorname{Pr}\left\{T_{\gamma, \phi}(\theta) \leq \chi_{1, \alpha}^{2}\left(1+3 n^{-1}\right)\right\}=1-\alpha+O\left(n^{-2}\right) .
$$


This theorem says that when we choose $\gamma=-1$ and $\phi=-1 \pm \frac{\sqrt{5}}{3}$, the nonparametric likelihood test based on $T_{\gamma, \phi}(\theta)$ using the adjusted critical value $\chi_{1, \alpha}^{2}\left(1+3 n^{-1}\right)$ provides a refinement to the order $O\left(n^{-2}\right)$ on the null rejection probability error. It should be noted that the empirical likelihood statistic (i.e., $T_{\gamma, \phi}(\theta)$ with $\gamma=\phi=-1$ ) is not Bartlett correctable because the fourth cumulant $\kappa_{\gamma, \phi}^{(4)}$ of the signed root does not vanish at the order of $O\left(n^{-4}\right)$ (see the proof of Theorem 8 in the web appendix). This is due to the fact that the empirical moments contained in the signed root $S_{\gamma, \phi}$ show different limiting behaviors under the infill asymptotics (compared to the i.i.d. case), and the dominant term of the fourth cumulant $\kappa_{\gamma, \phi}^{(4)}$ takes a different form. Note that the Bartlett factor $\left(1+3 n^{-1}\right)$ does not contain any unknown objects.

Finally, we drop the assumption of constant volatility and consider a more general setup. Although the computations are quite cumbersome, it is possible to estimate tuning constants $\hat{\gamma}$ and $\hat{\phi}$ such that the nonparametric likelihood statistic $T_{\hat{\gamma}, \hat{\phi}}(\theta)$ is Bartlett correctable. The second-order properties of the nonparametric likelihood statistic in the general case are presented in the following theorem.

Theorem 9. Suppose Assumptions $X$ and $H$ hold true. Then, for $\hat{\gamma}, \hat{\phi}$, and a (defined in Section A.7 of the web appendix), the nonparametric likelihood statistic $T_{\hat{\gamma}, \hat{\phi}}(\theta)$ is Bartlett correctable, i.e., conditionally on the path of $\sigma$,

$$
\operatorname{Pr}\left\{T_{\hat{\gamma}, \hat{\phi}}(\theta) \leq \chi_{1, \alpha}^{2}\left(1+a n^{-1}\right)\right\}=1-\alpha+O\left(n^{-2}\right)
$$

This theorem says that even for the general non-constant volatility case, the nonparametric likelihood statistic $T_{\hat{\gamma}, \hat{\phi}}(\theta)$ with the estimated tuning constants $\hat{\gamma}$ and $\hat{\phi}$ using the adjusted critical value $\chi_{1, \alpha}^{2}\left(1+a n^{-1}\right)$ provides a refinement to the order $O\left(n^{-2}\right)$ on the null rejection probability error. In the general case, the Bartlett factor $a$ can be estimated 
by the method of moments or wild bootstrap as in Gonçalves and Meddahi (2009). See Section A.7 of the web appendix for details on the computation of $\hat{\gamma}$ and $\hat{\phi}$, and estimation of $a$. For the one-sided test, Gonçalves and Meddahi (2009) used the bootstrap to obtain a second-order refinement result of the order $o\left(n^{-1 / 2}\right)$. In contrast, we consider the two-sided test and show that our Bartlett correction of the nonparametric likelihood statistic can yield a refinement to the order $O\left(n^{-2}\right)$.

Although it is beyond the scope of this paper, we conjecture that analogous higher-order refinement results can be established for more general cases. As we explained in Section 2.3, the key step to establish the Bartlett correction for the nonparametric likelihood statistic is to characterize the third and fourth order cumulants of its asymptotic signed root. The calculations of these cumulants become more involved under general setups, such as processes containing jumps and noise. Such calculations for cumulants become even more complicated for the noise robust inference discussed in Section 3.3, where the empirical likelihood statistic involves an additional tuning sequence $K$. This additional complication is analogous to the one in Kitamura (1997), who established the Bartlett correctability of the blocked empirical likelihood statistic for dependent data.

\section{Simulation}

This section conducts simulation studies to evaluate the finite sample properties of the empirical likelihood methods presented above.

5.1. Simulation 1: Benchmark case. We adopt simulation designs considered in Gonçalves and Meddahi (2009). In particular, we consider the stochastic volatility model

$$
d X_{t}=\mu_{t} d t+\sigma_{t}\left(\rho_{1} d W_{1 t}+\rho_{2} d W_{2 t}+\sqrt{1-\rho_{1}^{2}-\rho_{2}^{2}} d W_{3 t}\right)
$$


where $W_{1 t}, W_{2 t}$, and $W_{3 t}$ are independent standard Brownian motions. In addition to the constant volatility case, we consider two different models for the volatility process $\sigma_{t}$. The first model for $\sigma_{t}$ is the $\operatorname{GARCH}(1,1)$ diffusion: $d \sigma_{t}^{2}=0.035\left(0.636-\sigma_{t}^{2}\right) d t+0.144 \sigma_{t}^{2} d W_{1 t}$. The second model is the two-factor diffusion model: $\sigma_{t}=f\left(-1.2+0.04 \sigma_{1 t}^{2}+1.5 \sigma_{2 t}^{2}\right)$, where $d \sigma_{1 t}^{2}=-0.00137 \sigma_{1 t}^{2} d t+d W_{1 t}, d \sigma_{2 t}^{2}=-1.386 \sigma_{2 t}^{2} d t+\left(1+0.25 \sigma_{2 t}^{2}\right) d W_{2 t}$, and

$$
f(x)=\exp (x) \mathbb{I}\left\{x \leq x_{0}\right\}+x_{0}^{-1 / 2} \exp \left(x_{0}\right) \sqrt{x_{0}-x_{0}^{2}+x^{2}} \mathbb{I}\left\{x>x_{0}\right\}
$$

with $x_{0}=\log (1.5)$. In addition to the case of no drift and no leverage effect (i.e., $\left.\left(\mu_{t}, \rho_{1}, \rho_{2}\right)=(0,0,0)\right)$, we allow for drift and leverage effects by setting $\left(\mu_{t}, \rho_{1}, \rho_{2}\right)=$ $(0.0314,-0.576,0)$ for the $\operatorname{GARCH}(1,1)$ model, and $\left(\mu_{t}, \rho_{1}, \rho_{2}\right)=(0.030,-0.30,-0.30)$ for the two-factor diffusion model.

For these cases, we compare seven methods to construct two-sided $95 \%$ confidence intervals: (i) the Wald-type interval $\left(\mathrm{W}_{\text {level }}\right){ }^{2}$ (ii) the empirical likelihood (EL) in Theorem 2, (iii) the nonparametric likelihood (NL) with $\gamma=-1$ and $\phi=-1+\frac{\sqrt{5}}{3}$ in Theorem 7 , (iv) the Bartlett corrected nonparametric likelihood (BNL) with the Bartlett correction factor $1+3 / n$ in Theorem $8,(\mathrm{v})$ the logarithmic transform based Wald-type interval $\left(\mathrm{W}_{\log }\right)$, (vi) the wild bootstrap based on the two-point distribution proposed in Gonçalves and Meddahi (2009, Proposition 4.5) (Boot ${ }_{\mathrm{GM}}$ ), and (vii) the local gaussian bootstrap proposed by Hounyo (2018) with block sizes $M=1,4$, and 12 . $\left(\operatorname{Boot}_{\mathrm{H}}(M)\right){ }^{3}$

Tables 1 and 2 present the actual coverage rates of each confidence interval across 10,000 Monte Carlo replications for five different sample sizes: $n=1152,288,48,24$, and 12, which correspond to 1.25-minute, 5-minute, half-hour, 1-hour, and 2-hour returns, respectively. All methods tend to undercover, especially when the sample size $n$ is small

\footnotetext{
${ }^{2}$ The $100(1-\alpha) \%$ asymptotic Wald -type confidence interval is $\left[\hat{\theta} \pm z_{\alpha / 2} \sqrt{\hat{V} / n}\right]$, where $\hat{V}=\frac{2 n}{3} \sum_{i=1}^{n} r_{i}^{4}$.

${ }^{3}$ The bootstrap confidence intervals use 499 bootstrap replications for each Monte Carlo replication.
} 
(or sampling in a fixed time interval is not too frequent). However, we find that the performance of Hounyo's (2018) local gaussian bootstrap is excellent. The two-factor model implies overall larger coverage distortions than the GARCH(1,1) model.

We first compare the proposed nonparametric likelihood methods (EL, NL, and BNL) and the conventional Wald confidence interval $\left(\mathrm{W}_{\text {level }}\right)$ to illustrate the discussions in Section 2.3. Indeed, EL, NL, and BNL outperform $\mathrm{W}_{\text {level }}$ for all cases. Among these nonparametric likelihood methods, BNL outperforms even for stochastic volatility models with drift and leverage effects despite the fact that the Bartlett correction in Theorem 8 does not theoretically provide an asymptotic refinement under non-constant volatility.

We now compare the nonparametric likelihood methods (EL, NL, and BNL) with the bootstrap methods $\left(\right.$ Boot $_{\mathrm{GM}}$ and Boot $\left._{\mathrm{H}}\right)$ and the logarithmic transform based Wald-type interval $\left(\mathrm{W}_{\mathrm{log}}\right)$. For the $\mathrm{GARCH}(1,1)$ model, Boot $_{\mathrm{H}}$ by Hounyo $(2018)$ tends to be closer to the nominal level than the other methods, and our nonparametric likelihood methods show similar performance as Boot ${ }_{\mathrm{GM}}$ by Gonçalves and Meddahi (2009) and $\mathrm{W}_{\mathrm{log}}$. For the twofactor model, BNL, in particular, is favorably comparable with $\operatorname{Boot}_{\mathrm{GM}}$, Boot $\mathrm{H}_{\mathrm{H}}$, and $\mathrm{W}_{\log }$. In this case, we should note that the results of Boot $_{\mathrm{H}}$ may be sensitive to the choice of the block size $M$. Overall, for the benchmark case, the proposed nonparametric likelihood methods perform equally as well as Gonçalves and Meddahi's (2009) wild bootstrap and the logarithmic transform based Wald interval, but less satisfactory than Hounyo's (2018) local gaussian bootstrap. It is interesting to investigate whether Hounyo's (2018) local approach can be adapted to our empirical likelihood approach (e.g., construct an empirical likelihood by exploiting the local Gaussian framework of Mykland and Zhang (2009)).

As discussed in Section 2.3, the nonparametric likelihood confidence intervals are range preserving while the conventional Wald-type confidence interval $\left(\mathrm{W}_{\text {level }}\right)$ may contain negative values. To illustrate this point, we report the frequencies of negative left endpoints 
of the Wald-type confidence intervals in Table 3. This shows that the Wald-type intervals tend to contain negative values particularly for small sample sizes.

5.2. Simulation 2: Test for jump. In this subsection we evaluate the finite sample properties of the nonparametric likelihood test for the presence of jumps discussed in Section 3.4. We adopt the simulation design in Dovonon et al. (2017), and consider the two-factor diffusion model with diurnality effects:

$$
\begin{aligned}
d \log S_{t} & =\mu_{t} d t+\sigma_{u, t} \sigma_{t}\left(\rho_{1} d W_{1 t}+\rho_{2} d W_{2 t}+\sqrt{1-\rho_{1}^{2}-\rho_{2}^{2}} d W_{3 t}\right)+d J_{t}, \\
\sigma_{u, t} & =0.88929198+0.75 \exp (-10 t)+0.25 \exp (-10(1-t)), \quad \sigma_{t}=f\left(-1.2+0.04 \sigma_{1 t}^{2}+1.5 \sigma_{2 t}^{2}\right),
\end{aligned}
$$

where $d \sigma_{1 t}^{2}=-0.00137 \sigma_{1 t}^{2} d t+d W_{1 t}, d \sigma_{2 t}^{2}=-1.386 \sigma_{2 t}^{2} d t+\left(1+0.25 \sigma_{2 t}^{2}\right) d W_{2 t}$, and $f(\cdot)$ is defined in (5.1). The process $\sigma_{u, t}$ models the diurnal U-shaped pattern in intraday volatility. When $\sigma_{u, t}=1$ for $t \in[0,1]$, the return process reduces to the simple case of no diurnality effects. $J_{t}$ is a finite activity jump process modeled as a compound Poisson process with constant jump intensity $\lambda$ and random jump size distributed as $N\left(0, \sigma_{\text {jump }}^{2}\right)$. For the null hypothesis of no jump in the return process, we set $\sigma_{\text {jump }}^{2}=0$. For the alternative hypothesis, we set $\lambda=0.058$ and $\sigma_{\text {jump }}^{2}=1.7241$.

We compare four methods to test for jumps: (i) the Wald-type test (Wald), ${ }^{4}$ (ii) the (one-sided) empirical likelihood test (EL) presented after Theorem 6 using the tripower variation $\left(m=3\right.$ and $\left.p_{1}=p_{2}=p_{3}=2 / 3\right)$, (iii) the adjusted Wald-type test by Huang

\footnotetext{
${ }^{4}$ The Wald statistic is defined as $T_{n}=\sqrt{n}\left(R V_{n}-B V_{n}\right) / \sqrt{\hat{V}_{n}}$, where $R V_{n}=\sum_{i=1}^{n} r_{i}^{2}, B V_{n}=$ $\frac{1}{\mu_{1}^{2}} \sum_{i=2}^{n}\left|r_{i}\right|\left|r_{i-1}\right|$, and $\hat{V}_{n}=\left(\mu_{1}^{-4}+2 \mu_{1}^{-2}-5\right) \frac{n}{\mu_{4 / 3}^{3}} \sum_{i=3}^{n}\left|r_{i}\right|^{4 / 3}\left|r_{i-1}\right|^{4 / 3}\left|r_{i-2}\right|^{4 / 3}$. The test then rejects the null of no jump at a significance level of $\alpha$ when $T_{n}>z_{1-\alpha}$, where $z_{1-\alpha}$ is the $(1-\alpha)$-th quantile of the standard normal distribution.
} 
and Tauchen (2005) $\left(\right.$ Wald $\left._{\text {adj }}\right),{ }^{5}$ and (iv) the bootstrap test by Dovonon et al. (2017) with $L=5$ and $M=4$ (Boot) ${ }^{6}$

We consider five different sample sizes: $n=1152,576,288,96$, and 48, which correspond to 1.25-minute, 2.5-minute, 5-minute, 15-minute, and half-hour returns. All results are based on 5,000 Monte Carlo replications.

Table 4 reports the rejection frequencies of the jump tests under the null of no jump at the $5 \%$ nominal significance level for both cases with and without diurnally effects. The Wald-type tests (both Wald and Wald $_{\text {adj }}$ ) tend to over-reject for both cases especially when the sample size is small (i.e., sampling in a fixed time interval is less frequent). In all cases, EL shows better performance in terms of the null rejection frequencies. The rejection frequencies vary from $9.3 \%(n=1152)$ to $26.9 \%(n=48)$ for Wald, from $7.4 \%$ $(n=1152)$ to $17.2 \%(n=48)$ for Wald $_{\text {adj }}$, from $6.2 \%(n=1152)$ to $10.7 \%(n=48)$ for Boot, while they vary from $5.3 \%(n=1152)$ to $8.0 \%(n=48)$ for EL.

We also analyze the power properties of the proposed jump test under the alternative hypothesis. We compare the calibrated powers of the four tests above (i.e., the rejection frequencies of these tests where the critical values are given by the Monte Carlo $95 \%$ percentiles of the corresponding test statistics under the data generation process satisfying the null hypothesis). Table 5 shows that EL is slightly less powerful than the other methods. Since EL has better null rejection properties than the others, these power properties characterize a tradeoff between the size and power properties of the empirical likelihood test and other tests.

\footnotetext{
${ }^{5}$ The adjusted Wald-type statistic is defined as $\frac{\sqrt{n}\left(1-B V_{n} / R V_{n}\right)}{\left.\sqrt{\left(\mu_{1}^{-4}+2 \mu_{1}^{-2}-5\right) \max \left\{1, T Q_{n} / B V_{n}^{2}\right.}\right\}}$, where $T Q_{n}=$ $\frac{n}{\mu_{4 / 3}^{3}} \sum_{i=3}^{n}\left|r_{i}\right|^{4 / 3}\left|r_{i-1}\right|^{4 / 3}\left|r_{i-2}\right|^{4 / 3}$.

${ }^{6}$ The choice of $L=5$ is based on the recommendation of Dovonon et al. (2017). The results are not very sensitive to the choice of $M$ when $L=5$. In our simulations, the bootstrap test uses 499 bootstrap replications for each Monte Carlo replication.
} 
5.3. Simulation 3: Noise robust inference. In Section B of the web appendix, we present additional simulation results for the noise robust empirical likelihood statistic discussed in Section 3.3.

\section{REAL DATA EXAMPLE}

To mitigate microstructure noise, as suggested in Hansen and Lunde (2006) and Bandi and Russel (2008), we consider 5-minute data consisting of intra-day quotes of Alcoa, American Express, Baxter, Citigroup, Dow, Gilead, Goldman Sachs, Intel Corporation, Met, Microsoft, Nike, Pfizer, Verizon and Yahoo from January 2, 2001 to November 15, 2005, which corresponds to 1236 trading days.

Table 6 reports the percentage of days identified with jumps for the period under investigation. ${ }^{7}$ To this end, we consider four methods to test for jumps: (i) the Wald-type test (Wald), (ii) (signed root) empirical likelihood (EL), (iii) (signed root) nonparametric likelihood (NL) with $\gamma=-1$ and $\phi=-1+\frac{\sqrt{5}}{3}$, and the bootstrap approach (Boot) proposed in Dovonon, Goncalves, Hounyo, and Meddahi (2018). In line with the Monte Carlo findings, we note that the Wald test tends to over detect jumps. Indeed, the percentage of days identified with jumps is always larger than 19\%. EL, NL and Boot imply very similar empirical findings. Using nonparametric likelihood procedures, the percentage of days identified with jumps is always smaller than $11 \%$.

\section{Conclusion}

In this paper, we propose empirical likelihood-based methods for interval estimation and hypothesis testing of volatility measures using high frequency data. Our empirical likelihood approach is extended to be robust to the presence of jumps and microstructure

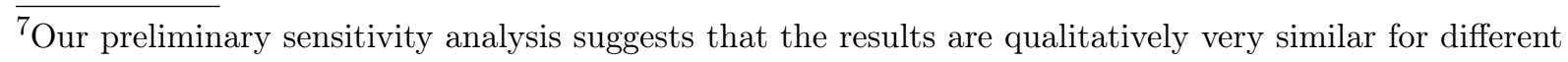
sampling frequencies. 
noise, and an empirical likelihood test to detect the presence of jumps is developed. We also investigate second-order properties of a general family of nonparametric likelihood statistics, and propose a method for Bartlett correction.

One important direction of future research is to extend our empirical likelihood approach to develop a new point estimator under over-identified estimating equations, where the number of estimating equations exceeds the number of parameters. Over-identified estimating equations naturally emerge in the present context if we combine different estimating equations for the same object of interest. In this case, the resulting maximum empirical likelihood estimator can be different from the existing estimators, and is expected to be more efficient. This extension is currently under investigation by the authors.

\section{Appendix A. TABLES}

\begin{tabular}{|c|c|c|c|c|c|c|c|c|c|}
\hline$n$ & $\mathrm{~W}_{\text {level }}$ & EL & NL & BNL & $\mathrm{W}_{\log }$ & Boot $_{\mathrm{GM}}$ & $\operatorname{Boot}_{H}(1)$ & $\operatorname{Boot}_{H}(4)$ & $\operatorname{Boot}_{H}(12)$ \\
\hline \multicolumn{10}{|c|}{ constant volatility } \\
\hline 12 & 85.78 & 88.06 & 87.62 & 90.36 & 90.28 & 86.62 & 94.26 & 94.40 & 94.32 \\
\hline 24 & 89.20 & 91.70 & 91.28 & 92.72 & 92.20 & 91.72 & 94.22 & 94.80 & 94.54 \\
\hline 48 & 91.80 & 93.68 & 93.38 & 94.26 & 93.88 & 93.56 & 94.00 & 94.60 & 94.58 \\
\hline 288 & 94.28 & 94.72 & 94.70 & 94.84 & 94.64 & 94.34 & 94.58 & 94.78 & 95.04 \\
\hline 1152 & 94.90 & 94.90 & 94.90 & 94.90 & 95.54 & 95.18 & 94.18 & 95.30 & 94.96 \\
\hline \multicolumn{10}{|c|}{ GARCH $(1,1)$ diffusion } \\
\hline 12 & 86.06 & 88.64 & 88.28 & 90.64 & 89.76 & 87.16 & 93.52 & 94.22 & 94.34 \\
\hline 24 & 90.38 & 92.44 & 91.90 & 93.46 & 92.66 & 92.12 & 93.46 & 94.80 & 94.52 \\
\hline 48 & 92.46 & 93.88 & 93.70 & 94.52 & 92.96 & 93.96 & 93.84 & 94.20 & 94.38 \\
\hline 288 & 94.04 & 94.70 & 94.62 & 94.80 & 94.66 & 93.92 & 93.92 & 94.22 & 94.04 \\
\hline 1152 & 94.94 & 95.06 & 95.06 & 95.06 & 94.98 & 94.66 & 94.78 & 94.56 & 95.22 \\
\hline \multicolumn{10}{|c|}{ Two-factor diffusion } \\
\hline 12 & 79.86 & 82.96 & 82.30 & 85.76 & 86.44 & 80.16 & 93.22 & 91.24 & 87.16 \\
\hline 24 & 85.06 & 88.66 & 88.22 & 90.18 & 88.96 & 87.50 & 93.26 & 92.40 & 88.70 \\
\hline 48 & 88.50 & 91.34 & 91.08 & 91.84 & 90.90 & 90.68 & 93.56 & 92.60 & 90.50 \\
\hline 288 & 93.38 & 94.18 & 93.98 & 94.16 & 94.36 & 93.52 & 93.62 & 95.06 & 93.90 \\
\hline 1152 & 94.50 & 94.56 & 94.48 & 94.48 & 95.00 & 93.90 & 93.92 & 94.76 & 94.92 \\
\hline
\end{tabular}

TABLE 1. Coverage probabilities of nominal 95\% confidence intervals for integrated volatility (no leverage and no drift case) 


\begin{tabular}{|c|c|c|c|c|c|c|c|c|c|}
\hline$n$ & $\mathrm{~W}_{\text {level }}$ & $\overline{E L}$ & NL & $\overline{\mathrm{BNL}}$ & $W_{\log }$ & Boot $_{\mathrm{GM}}$ & $\operatorname{Boot}_{\mathrm{H}}(1)$ & $\operatorname{Boot}_{H}(4)$ & $\operatorname{Boot}_{H}(12)$ \\
\hline & \multicolumn{9}{|c|}{ GARCH $(1,1)$ diffusion } \\
\hline 12 & 85.84 & 88.60 & 88.02 & 90.50 & 89.78 & 87.02 & 93.96 & 94.56 & 94.42 \\
\hline 24 & 89.02 & 91.06 & 90.64 & 92.26 & 92.08 & 92.40 & 94.44 & 94.08 & 94.44 \\
\hline 48 & 92.14 & 93.70 & 93.58 & 94.16 & 93.38 & 93.40 & 94.52 & 94.64 & 94.18 \\
\hline 288 & 95.20 & 95.48 & 95.42 & 95.50 & 94.48 & 94.80 & 94.56 & 94.32 & 95.42 \\
\hline \multirow[t]{2}{*}{1152} & 95.14 & 95.00 & 94.94 & 94.98 & 94.86 & 94.46 & 94.60 & 94.00 & 94.14 \\
\hline & \multicolumn{9}{|c|}{ Two-factor diffusion } \\
\hline 12 & 79.76 & 84.34 & 83.86 & 86.70 & 86.84 & 79.60 & 94.36 & 91.28 & 87.72 \\
\hline 24 & 84.68 & 88.34 & 88.10 & 89.44 & 88.90 & 87.16 & 94.46 & 91.88 & 89.46 \\
\hline 48 & 88.04 & 91.08 & 90.80 & 91.68 & 91.04 & 90.32 & 92.96 & 92.74 & 91.56 \\
\hline 288 & 93.18 & 94.10 & 94.02 & 94.12 & 94.10 & 93.86 & 93.78 & 93.24 & 93.04 \\
\hline 1152 & 94.56 & 95.46 & 95.38 & 95.42 & 94.54 & 94.16 & 94.72 & 94.74 & 93.90 \\
\hline
\end{tabular}

TABLE 2. Coverage probabilities of nominal $95 \%$ confidence intervals for integrated volatility (with leverage and drift)

\begin{tabular}{|c|c|c|c|c|c|c|}
\hline & $95 \%$ & $99 \%$ & $99.9 \%$ & $95 \%$ & $99 \%$ & $99.9 \%$ \\
\hline$n$ & GARC & $\mathrm{H}(1,1)$ & diffusion & \multicolumn{3}{|c|}{ Two-factor diffusion } \\
\hline 12 & 80.64 & 99.85 & 100 & 91.86 & 99.96 & 100 \\
\hline 24 & 6.94 & 43.60 & 95.44 & 40.61 & 79.32 & 99.02 \\
\hline 48 & 0.01 & 0.47 & 7.21 & 8.75 & 25.59 & 58.14 \\
\hline 288 & 0 & 0 & 0 & 0 & 0.04 & 0.23 \\
\hline 1152 & 0 & 0 & 0 & 0 & 0 & 0 \\
\hline
\end{tabular}

TABLE 3. Frequencies (measured by percentages) of negative left endpoints of $95 \%, 99 \%$, and $99.9 \%$ Wald confidence intervals for integrated volatility (with leverage and drift)

\begin{tabular}{rcccccccc}
\hline$n$ & Wald & EL & Wald $_{\text {adj }}$ & Boot & Wald & EL & Wald $_{\text {adj }}$ & Boot \\
\hline \multicolumn{4}{c}{ without diurnal effects } & \multicolumn{4}{c}{ with diurnal effects } \\
48 & 22.4 & 8.0 & 14.6 & 8.5 & 26.9 & 7.3 & 17.2 & 10.7 \\
96 & 15.1 & 5.7 & 9.9 & 7.3 & 18.8 & 5.4 & 13.3 & 8.9 \\
288 & 11.2 & 4.9 & 8.3 & 6.2 & 12.0 & 4.9 & 9.6 & 7.4 \\
576 & 10.4 & 5.7 & 8.3 & 6.4 & 11.7 & 5.7 & 9.7 & 7.1 \\
1152 & 9.3 & 5.3 & 7.4 & 6.3 & 9.5 & 5.4 & 7.8 & 6.2 \\
\hline
\end{tabular}

TABLE 4. Rejection frequencies of jump tests at $5 \%$ level 


\begin{tabular}{rcccccccc}
\hline$n$ & Wald & EL & Wald $_{\text {adj }}$ & Boot & Wald & EL & Wald $_{\text {adj }}$ & Boot \\
\hline \multicolumn{4}{c}{ without diurnal effects } & \multicolumn{5}{c}{ with diurnal effects } \\
48 & 74.9 & 66.4 & 75.2 & 74.9 & 72.8 & 65.0 & 72.1 & 72.8 \\
96 & 82.6 & 77.0 & 82.6 & 82.6 & 79.8 & 74.9 & 79.5 & 79.8 \\
288 & 86.9 & 83.4 & 86.9 & 86.9 & 83.7 & 80.9 & 83.7 & 83.7 \\
576 & 89.7 & 85.9 & 89.4 & 89.7 & 87.6 & 83.0 & 87.6 & 87.6 \\
1152 & 89.7 & 85.9 & 89.4 & 89.7 & 87.6 & 84.5 & 87.6 & 87.6 \\
\hline
\end{tabular}

TABle 5. Calibrated power of jump tests

\begin{tabular}{lllrr}
\hline & Wald & EL & \multicolumn{1}{c}{ NL } & Boot \\
\hline Alcoa & 20.54 & 5.65 & 6.47 & 5.44 \\
American Express & 19.62 & 6.76 & 7.52 & 6.32 \\
Baxter & 23.67 & 8.13 & 8.76 & 7.22 \\
Citigroup & 21.69 & 8.92 & 9.37 & 8.88 \\
Dow & 23.07 & 7.76 & 8.12 & 7.11 \\
Gilead & 21.02 & 6.16 & 7.02 & 6.01 \\
Goldman Sachs & 19.76 & 4.87 & 5.24 & 4.67 \\
Intel Corporation & 21.11 & 7.34 & 7.61 & 6.85 \\
Met & 22.65 & 9.42 & 10.26 & 9.13 \\
Microsoft & 22.02 & 8.14 & 9.02 & 8.04 \\
Nike & 21.97 & 7.54 & 8.02 & 7.08 \\
Pfizer & 21.65 & 9.59 & 10.15 & 8.93 \\
Verizon & 22.15 & 7.06 & 7.56 & 6.84 \\
Yahoo & 22.87 & 8.05 & 9.23 & 7.84 \\
\hline
\end{tabular}

TABLE 6. Percentage of days identified with jumps for the period from January 2, 2001 to November 15, 2005.

\section{REFERENCES}

[1] Aït-Sahalia, Y. and J. Jacod (2009a) Testing for jumps in a discretely observed process, Annals of Statistics, 37, 184-222.

[2] Aït-Sahalia, Y. and J. Jacod (2009b) Estimating the degree of activity of jumps in high frequency data, Annals of Statistics, 37, 2202-2244. 
[3] Aït-Sahalia, Y. and J. Jacod (2014) High-Frequency Financial Econometrics, Princeton University Press.

[4] Aït-Sahalia, Y., Mykland, P. A. and L. Zhang (2011) Ultra high frequency volatility estimation with dependent microstructure noise, Journal of Econometrics, 160, 160-175.

[5] Andersen, T. G., Dobrev, D. and E. Schaumburg (2012) Jump-robust volatility estimation using nearest neighbor truncation, Journal of Econometrics, 169, 75-93.

[6] Baggerly, K. A. (1998) Empirical likelihood as a goodness-of-fit measure, Biometrika, 85, 535-547.

[7] Bandi, F. M. and J. R. Russell (2008) Microstructure noise, realized variance, and optimal sampling, Review of Economic Studies, 75, 339-369.

[8] Barndorff-Nielsen, O. E., Graversen, S. E., Jacod, J. and N. Shephard (2006) Limit theorems for bipower variation in financial econometrics, Econometric Theory, 22, 677-719.

[9] Barndorff-Nielsen, O. E., Hansen, P. R., Lunde, A. and N. Shephard (2008) Designing realized kernels to measure the ex post variation of equity prices in the presence of noise, Econometrica, 76, 1481-1536.

[10] Barndorff-Nielsen, O. E. and N. Shephard (2002) Econometric analysis of realized volatility and its use in estimating stochastic volatility models, Journal of the Royal Statistical Society, B, 64, 253-280.

[11] Barndorff-Nielsen, O. E. and N. Shephard (2004) Power and bipower variation with stochastic volatility and jumps, Journal of Financial Econometrics, 2, 1-37.

[12] Barndorff-Nielsen, O. E. and N. Shephard (2005). How accurate is the asymptotic approximation to the distribution of realised variance?, in Andrews, D. W. K. and J. H. Stock (eds.), Identification and Inference for Econometric Models, Cambridge University Press, 306-331.

[13] Barndorff-Nielsen, O. E. and N. Shephard (2006) Econometrics of testing for jumps in financial economics using bipower variation, Journal of Financial Econometrics, 4, 1-30.

[14] Barndorff-Nielsen, O. E., Shephard, N. and M. Winkel (2006) Limit theorems for multipower variation in the presence of jumps, Stochastic Processes and their Applications, 116, 796-806.

[15] Choi, S., Hall, W. J. and A. Schick (1996) Asymptotically uniformly most powerful tests in parametric and semiparametric models, Annals of Statistics, 24, 841-861.

[16] Cressie, N. and T. R. C. Read (1984) Multinomial goodness-of-fit tests, Journal of the Royal Statistical Society, B, 46, 440-464. 
[17] DiCiccio, T. J., Hall, P. and J. Romano (1991) Empirical likelihood is Bartlett-correctable, Annals of Statistics, 19, 1053-1061.

[18] Dumitru, A.-M. and G. Urga (2012) Identifying jumps in financial assets: A comparison between nonparametric jump tests, Journal Business 83 Economic Statistics, 30, 242-255.

[19] Dovonon, P., Gonçalves, S. and N. Meddahi (2013) Bootstrapping realized multivariate volatility measures, Journal of Econometrics, 172, 49-65.

[20] Dovonon, P., Gonçalves, S., Hounyo, U. and N. Meddahi (2017) Bootstrapping high-frequency jump tests, forthcoming in Journal of the American Statistical Association.

[21] Gonçalves, S. and N. Meddahi (2008) Edgeworth corrections for realized volatility, Econometric Reviews, 27, 139-162.

[22] Gonçalves, S. and N. Meddahi (2009) Bootstrapping realized volatility, Econometrica, 77, 283-306.

[23] Gonçalves, S. and N. Meddahi (2011). Box-Cox transforms for realized volatility, Journal of Econometrics, 160, 129-144.

[24] Hansen, P. R. and A. Lunde (2006), Realized variance and market microstructure noise, Journal of Business \&3 Economic Statistics, 24, 127-161.

[25] Hall, P. and B. La Scala (1990) Methodology and algorithms of empirical likelihood, International Statistical Review, 58, 109-127.

[26] Heston, S. L. (1993) A closed-form solution for options with stochastic volatility with applications to bond and currency options, Review of Financial Studies, 6, 327-343.

[27] Hjort, N. L., McKeague, I. W. and I. van Keilegom (2009) Extending the scope of empirical likelihood, Annals of Statistics, 37, 1079-1111.

[28] Hounyo, U. (2018) A local Gaussian bootstrap method for realized volatility and related beta, forthcoming in Econometric Theory.

[29] Hounyo, U., Gonçalves, S. and N. Meddahi (2017) Bootstrapping pre-averaged realized volatility under market microstructure noise, Econometric Theory, 33, 791-838.

[30] Hounyo, U. and B. Veliyev (2016) Validity of Edgeworth expansions for realized volatility estimators, Econometrics Journal, 19, 1-32.

[31] Huang, X. and G. Tauchen (2005) The relative contribution of jumps to total price variance, Journal of Financial Econometrics, 3, 456-499. 
[32] Jacod, J., Li, Y., Mykland, P. A., Podolskij, M. and M. Vetter (2009) Microstructure noise in the continuous case: the pre-averaging approach, Stochastic Processes and their Applications, 119, 2249-2276.

[33] Jacod, J. and P. Protter (1998) Asymptotic error distributions for the Euler method for stochastic differential equations, Annals of Probability, 26, 267-307.

[34] Jacod, J. and M. Rosenbaum (2013) Quarticity and other functionals of volatility: Efficient estimation, Annals of Statistics, 41, 1462-1484.

[35] Kong, X.-B. (2012) Confidence interval of the jump activity index based on empirical likelihood using high frequency data, Journal of Statistical Planning and Inference, 142, 1378-1387.

[36] Kitamura, Y. (1997) Empirical likelihood methods with weakly dependent process, Annals of Statistics, 25, 2084-2102.

[37] Li, Y., Zhang, Z. and X. Zheng (2013) Volatility inference in the presence of both endogenous time and microstructure noise, Stochastic Processes and their Applications, 123, 2696-2727.

[38] Mykland, P. A. and L. Zhang (2009) Inference for continuous semimartingales observed at high frequency, Econometrica, 77, 1403-1445.

[39] Owen, A. B. (1988) Empirical likelihood ratio confidence intervals for a single functional, Biometrika, $75,237-249$.

[40] Owen, A. B. (2001) Empirical Likelihood, Chapman and Hall/CRC.

[41] Podolskij, M. and N. Yoshida (2016) Edgeworth expansion for functionals of continuous diffusion processes, Annals of Applied Probability, 26, 3415-3455.

[42] Renault, E., Sarisoy, C. and B. J. M. Werker (2017) Efficient estimation of integrated volatility and related processes, Econometric Theory, 33, 439-478.

[43] Schennach, S. M. (2005) Bayesian exponentially tilted empirical likelihood, Biometrika, 92, 31-46.

[44] Schennach, S. M. (2007) Point estimation with exponentially tilted empirical likelihood, Annals of Statistics, 35, 634-672.

[45] Zhang, L., Mykland, P. A. and Y. Aït-Sahalia (2005) A tale of two time scales: determining integrated volatility with noisy high-frequency data, Journal of the American Statistical Association, 100, 13941411. 
[46] Zhang, L., Mykland, P. A. and Y. Aït-Sahalia (2011) Edgeworth expansions for realized volatility and related estimators, Journal of Econometrics, 160, 190-203

University of Applied Sciences and Arts of Italian Switzerland, Galleria 2 - 100 Via Cantonale 6928, Manno, CH

E-mail address: lorenzo.camponovo@supsi.ch

Graduate School of Economics, Hitotsubashi University, 2-1 Naka, Kunitachi, Tokyo 186-8601, JAPAN.

E-mail address: matsushita.y@r.hit-u.ac.jp

Department of Economics, London School of Economics, Houghton Street, London, WC2A 2AE, UK.

E-mail address: t.otsu@lse.ac.uk 